\title{
Characterization of the Ground-to-Air Ranging Performance of the 960-1215 MHz ARNS Band Using OFDM Measurements in the 902-928 MHz ISM Band
}

\author{
Okuary Osechas $^{1}$, Nicolas Schneckenburger ${ }^{1}$, Wouter Pelgrum $^{2}$, Elisabeth Nossek $^{1}$, and Michael \\ Meurer $^{1}$ \\ ${ }^{1}$ German Aerospace Center (DLR), Oberpfaffenhofen, Germany \\ ${ }^{2}$ Ohio University, Athens, OH
}

\begin{abstract}
In this paper we discuss the feasibility of prototyping radionavigation hardware, designed to operate in the ARNS band, by flight testing it in the $902-928 \mathrm{MHz}$ ISM band. A central challenge in this effort was the shared nature of the ISM band, which causes high in-band interference levels and, consequently, a reduction in the signal-to-noise ratio. We propose mitigating strategies that enable operation in the ISM band, as well as a methodology for assessing the equivalence of results with ARNS band measurements.
\end{abstract}

\section{Introduction}

In the effort to provide Alternative Position, Navigation, and Timing (APNT) systems, many future and existing options involve signals in the Aeronautical Radio Navigation Service (ARNS) band. In order to obtain a valid, meaningful statistical model of any APNT system, it needs to be characterized with flight experiments. However, regulatory constraints significantly hinder flight testing ARNS-band systems. One band adjacent to the ARNS band is the Industrial, Medical, and Scientific (ISM) band located between 902 and $928 \mathrm{MHz}$. We propose that one possible solution to the conundrum of testing ARNS band systems is to resort to flight tests that use the ISM band.

As a test for the proposed solution we assess the performance of a system based on Orthogonal Frequency-Division Multiplexing (OFDM) in the ISM band, using flight-test data gathered in the Summer of 2015 at Ohio University. We compare the ISM band data with data gathered from Distance Measuring Equipment (DME) hardware in prior experiences [1, 2.

The procedure by which two data sets can be compared is depicted in figure 1. The comparison, which happens in terms of ranging performance, checks the consistency of the collected data with data available from previous flight testing. We show how the new data fits in with the older data in a way that supports claim that the two frequency bands are similar enough to each other to support prototyping of ARNS band hardware in the ISM band.

This research was conducted under the hypothesis that propagation in the ISM band is expected to be statistically similar to the ARNS band, but momentarily different because of the differing signal phase relationships. The time-varying nature of the channel is expected to lead to statistically equivalent channel properties in both bands.

The equivalence of the two frequency bands, in terms of DME ranging errors, can be tested by checking the consistency of the performance prediction with information available from prior flight experiences [1]. In checking the consistency between the two data sets we exploit the spatial information available for the DME ranging errors. The flight experiments were designed specifically to study gaps in the coverage of existing range error data. The spatial distribution of predicted ranging errors (from ISM band data) fits in well with the existing data, as discussed in the results section. 


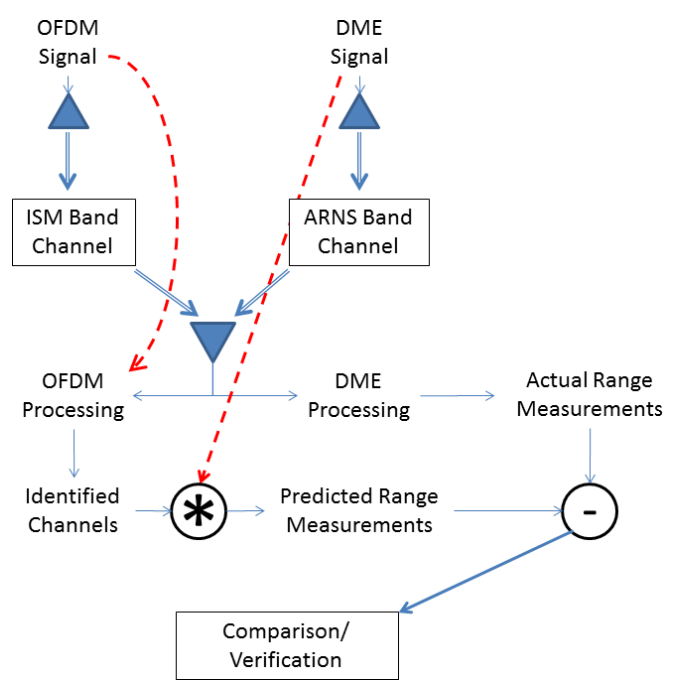

Figure 1: Comparing the DME ranging performance in the ISM band with that of the ARNS band. The OFDM signal, in the ISM band, is used to estimate the channel impulse response at any given point in time. That channel impulse response is used to predict DME ranging performance and compare the result with the actually measured ranging performance of DME.

\section{Methodology}

Characterizing ranging in the ISM Band, as well as the L Band, requires flight tests with hardware able to handle both bands. The method is described in detail in [3, which discusses channel characterization in great depth. For the purposes of this paper, we take channel characterization for granted and focus on the ranging performance obtainable from predicted and measured DME errors, as transmitted in the ISM and ARNS bands. A technical point to note is that the DME range measurements from the flight campaign in August 2015 were not yet available at the time of writing and the consistency of prediction with reality was done by comparing behavior at a given location with the behavior at nearby, previously characterized locations.

Our setup uses a wide-band OFDM signal in the ISM band to characterize the air-to-ground radio channel. We use that information to predict the ranging error characteristic for a DME signal (in the ARNS band). The resulting prediction is compared to the ranging errors measured in previous flight experiments.

To make the results in the two frequency bands comparable, we resort to assessing the ranging performance, as an indicator of the potential of the radio channel to support navigation services.

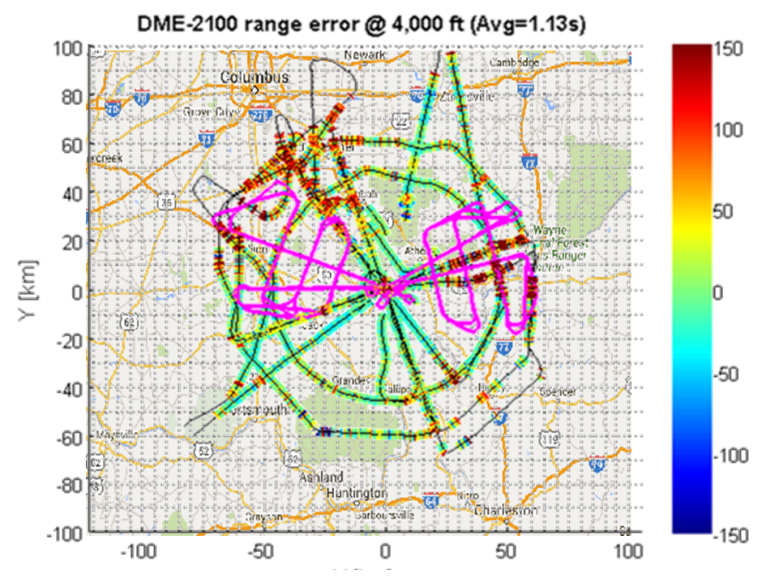

Figure 2: The flight paths for both sorties, pictured in magenta, were designed to facilitate the contrast between areas with expectations of DME strong ranging errors, with areas where DME errors were expected to be milder. Each flight has one "dirty" and one "clean" radial portion, as well as transverse sections.

The ranging performance is computed from the channel impulse response, by convolving the ranging waveform (i.e. DME) with the channel impulse response and measuring the delay of the resulting signal.

The flight paths were partly designed to cover locations previously not charted for DME ranging error. In addition each flight path was designed to have two radial sections, where one was surrounded with areas known to have high ranging errors ("dirty" radial) and the other was surrounded by points with much smaller errors ("clean" radial). For each flight, several portions of orbitals were also included in the flight path, with the expectation that the orbitals further away from the base would have stronger errors than those closer to the base.

\section{Hardware}

The airborne set-up included all the hardware necessary to broadcast an OFDM signal in the ISM band and a fully operational DME system. An arbitrary waveform generator was configured to broadcast an OFDM signal optimized for channel characterization, as described in [3, which was amplified and broadcast through a dedicated L-band antenna.

The ground set-up was shared with a DME ground station, which required diplexing the ISM-band signal and the ARNS-band signal. The two signals shared the antenna on the ground, which output it ISM band signal to a diplexer. The resulting ISM band signal 


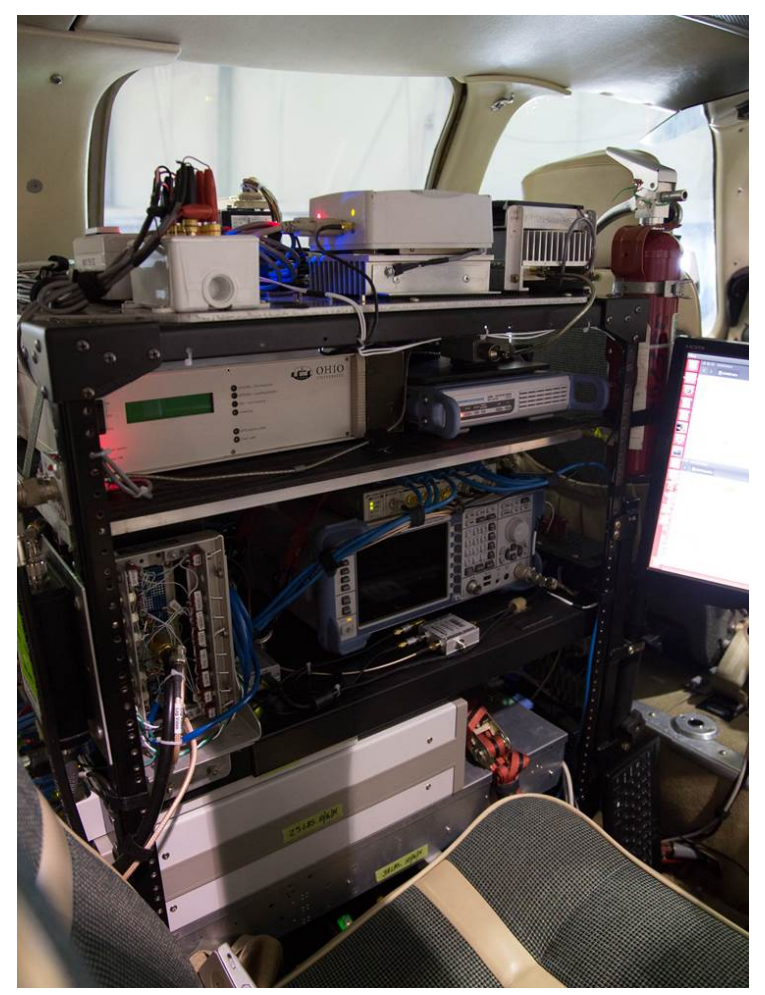

Figure 3: The ground side receives the OFDM signal and a DME interrogator signal; it also transmits DME replies through the same antenna. In addition, a GNSS antenna receives satellite signals that are used to compute an RTK-based ground truth, this enables precise synchronization with the airborne system. DME response signals are generated using a USRP, also the decoding of both DME and OFDM signals is done in the USRP. The ground setup also generates virtual DME traffic, to simulate DME reception in a crowded airspace.

went to a software-defined receiver, which sampeled the incoming RF signal at 12.5 megasamples per second.

In addition to the DME setup and the channel sounding hardware, both the aircraft and ground station were equipped with a truth system, based on satellite navigation hardware, to compute reference trajectories and synchronize the clocks of both installations. For details on the method, please refer to [3].

\section{Signals}

The setup requires signals in two different frequency bands: a standard DME signal in the ARNS band and a channel sounding signal in the ISM band. The channel sounding signal has a much higher bandwidth

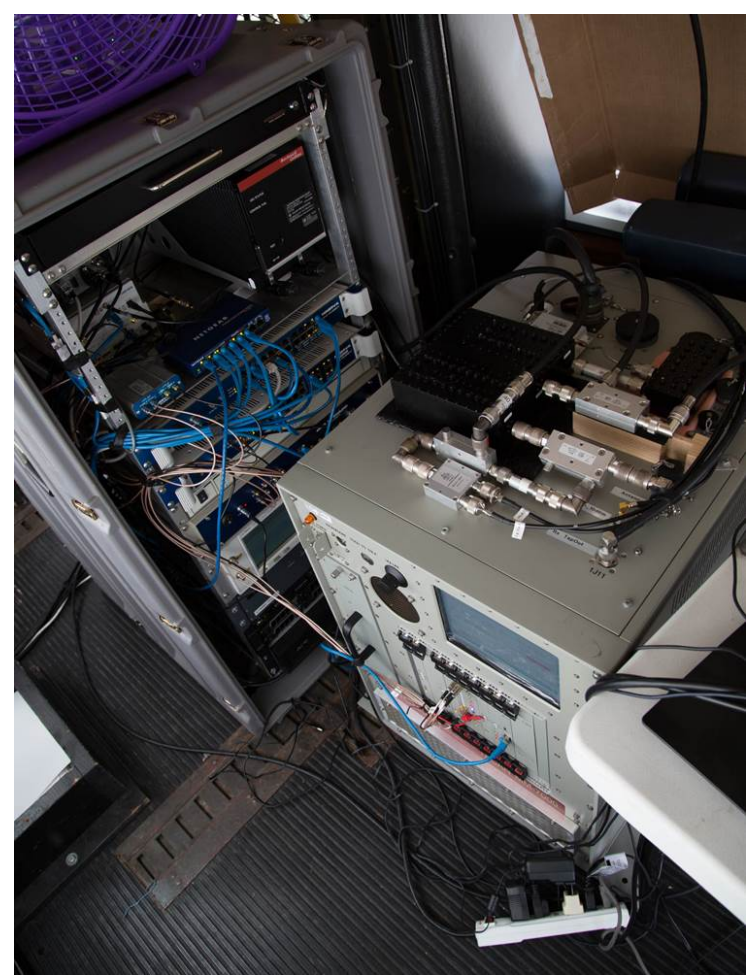

Figure 4: The airborne side produces DME interrogations and the OFDM channel sounding signal and receives DME replies from the ground station. A GNSS receiver distributes a 1 pulse per second synchronization signal to the on-board system, but it is also used for synchronization with the ground setup.

than the DME signal and, as such, the ranging performance attainable by that signal itself does not meaningfully compare with the ranging performance of the DME signal. In this sense, the comparison between two frequency bands needs a different figure of merit.

One option is to assess the ranging performance of the real DME signal with the predicted performance of a DME signal, after the ISM band channel parameters have been estimated. A second potential approach is to correlate normalized ranging errors for both signals against each other.

The DME signal consists of a Gaussian-shaped double pulse envelope and is well described in [4]; for the purposes of this paper it is only relevant that the DME signal is centered at $1107 \mathrm{MHz}$ and has a bandwidth of $0.5 \mathrm{MHz}$.

For channel sounding we use an OFDM-based signal optimized for low peak-to-average power ratio (PAPR) 5] and uniform frequency spectrum, this 
waveform is in accordance with the method of 6. For this set of experiments the channel sounding signal was centered at $915 \mathrm{MHz}$, with a bandwidth of $10 \mathrm{MHz}$. The autocorrelation functions of the baseband version of these two signals are shown in figure 5 .

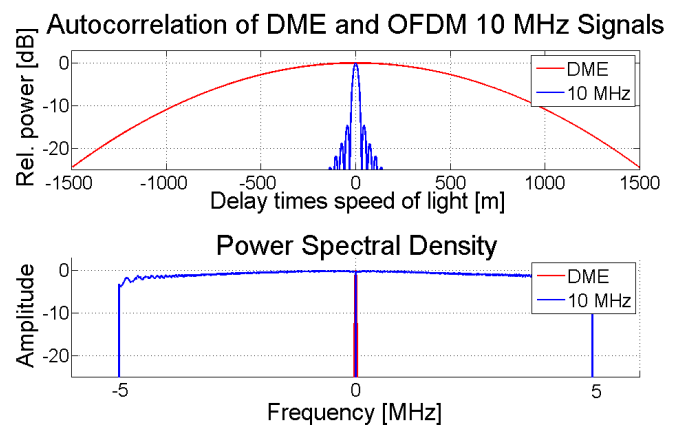

Figure 5: Comparison of the two signals. The autocorrelation (top) shows the finer time resolution achievable with the $10 \mathrm{MHz}$ channel sounding signal, as the DME signal spreads over a much longer range. In the frequency domain (bottom) the channel sounding signal has a much more uniform distribution than the DME signal, in addition to a larger bandwidth.

An important feature of the two autocorrelation functions plotted in figure 5 is that the time resolution of the OFDM signal is much higher than that of the DME signal; this is a consequence of the higher bandwidth available for the OFDM signal. This higher time resolution is necessary to use the computed channel transfer functions as a means of predicting the DME ranging error.

In our setup, the OFDM signal is broadcast from the aircraft and received at the ground station. Since the waveform is known and the time of transmission is synchronized between air and ground, using GNSSbased time stamping as described above, the received signal can be deconvolved with the transmit signal to obtain an estimate of the channel transfer function or impulse response.

\section{Flight Path Design}

From the data gathered from prior flight experiments [1] and from the visual inspection of panoramic images taken from the location of the ground station as presented in 2, we identified angular sections around the ground station where strong ranging errors are to be expected. Specifically, two sectors of interest go from 70 and 95 degrees and from 275 and 355 degrees. These sectors correspond to areas where

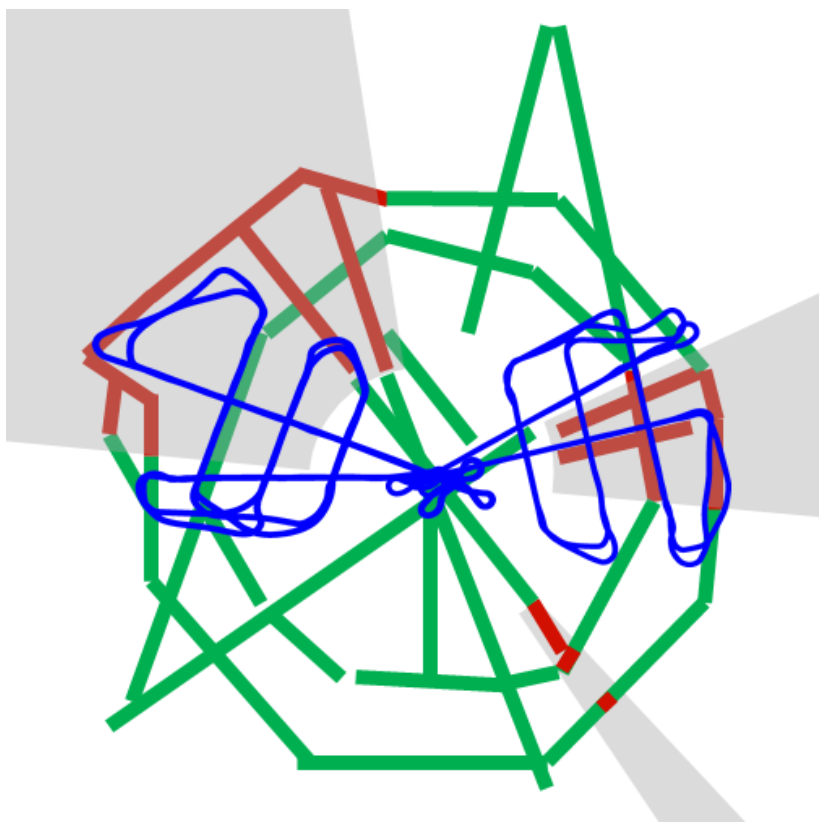

Figure 6: The spatial correlation of ranging errors can be described in terms of angular sections in which stronger ranging errors are expected. From older test data [1] this image simplifies the data of figure 2. Green lines indicate smaller measured ranging errors, red lines indicate stronger ranging errors. The areas indicated in gray are extrapolations of the prior data, where strong ranging errors are expected, given the error scenario of figure 8 . The traces in blue indicate the flight paths that resulted from the considerations derived from this fault model, each flight consisting of two radial sections, one going through the gray shaded areas and one avoiding those areas.

trees and other environmental features obstruct the horizon [2].

Based on the occlusion model shown in figure 8 we derive areas (or "slices") in which high ranging errors are expected, based on prior flight data. In figure 6 we show a simplified version of the ranging performance computed for figure 2 with smaller ranging errors indicated in green and stronger errors in red.

Figures 2 and 6 show the flight paths for the two sorties considered in this paper, one oriented towards the east of the ground station, the other towards the west. In both cases one radial aligns with an area of strong errors (dirty radial), the other going through an area of less errors (clean radial). The dirty radial of the east-bound flight was set at 80 degrees, which is inside the sector of ranging errors between 70 and 95 degrees, a the clean radial was set 
just to the north of that sector at 65 degrees. For the westward flight we chose a dirty radial at 300 degrees, while the clean portion was set to 270 degrees.

The flight paths were designed in a way that the results would unequivocally show whether the predicted DME errors resemble the measured DME errors or not. Predicted errors along the flight paths should, therefore, exhibit a clean radial and a dirty radial, if they support hypothesis that testing in the ISM band can be used in the design of ARNS band hardware.

\section{Predicting DME Errors}

While it is possible to use the ISM band signal to provide range measurements, the bandwidth of the signal is so much higher than that of DME that the ranging errors will be much smaller than those of DME, simply because the signal has a much higher resolution in time than the DME signal would. For a discussion of this observation, please refer to figure 5

Predicting DME ranging errors, based on measuring the received ISM band signal requires estimating the impulse response of the radio channel. As indicated in figure 1 the impulse response is computed by deconvolving an ideal DME waveform from the received ISM band signal. The resulting impulse response consists of a set of complex weights that model the LoS and echoes that would be received at the ground station, if the transmit signal were an ideal impulse.

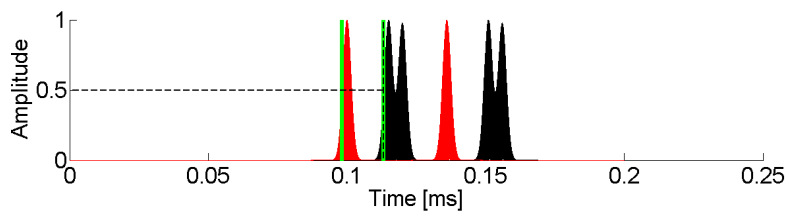

Figure 7: The DME signal consists of two Gaussian pulses, modulated with a carrier at $1107 \mathrm{MHz}$ (red). The received signal will typically contain a LoS component and one or more echoes (black). The time localization of a pulse is often defined as the point at which the pulse reaches half the maximum amplitude. The green bars indicate the time transmission time and the receive time that enables the range measurement.

The converse process can be applied to an ideal DME signal to determine what the receive waveform would be, should the transmit signal in the ISM band be DME-like, instead of the OFDM signal. This can be accomplished by summing delayed, attenuated and frequency-shifted copies of an ideal DME signal, corresponding to the LoS signal and echoes identified from the deconvolution.

By thus predicting the received waveform for a hypothetical DME transmission in the ISM band, we can then predict the ranging measurement that would obtained through such a channel. Knowing the waveform makes it possible to apply a DME detection algorithm and predict the ranging error associated with the identified radio channel, as indicated in figure 7 .

\section{Comparing Predicted and Measured DME Errors}

With the available data, one option for verifying the comparability of ISM and ARNS band propagation is to exploit the spatial correlation of DME errors. We propose a model that allows assessing the plausibility and consistence of the ranging predictions. We model the ranging error on the existing data as sections in azimuth and range, hereby distinguishing sections where ranging errors are relatively strong from sections where the ranging errors are relatively mild.

Modeling the error as angular slices is equivalent to attributing ranging errors to an occlusion or attenuation of the LoS, as suggested in [2]. For a given altitude and azimuth angle an occluded LoS remains occluded if the user moves away from the ranging source. The occlusion leads to an increase in the relative power of non-line-of-sight (NLOS) components compared to the LoS signal, which in turn can lead to ranging errors.

In scenario where the relative power of the NLOS components become sufficiently strong that the LoS power is negligible by comparison, the measured distance between transmitter and receiver is falsified. In that case the first signal to be detected at the receiver is not the LoS (as it is occluded), but a reflected signal, which by definition travels a longer distance.

Any occlusion of the LoS that spans a finite range in azimuth angles leads to ranging errors that correlate over angular sectors, breaking up the area around the ground station into "slices" (figure 8).

For the characterization of the ranging error around a terrestrial ranging source, the surrounding area may be broken up into slices. Of these slices, some will have stronger ranging errors than others, depending on the availability of a LoS and on the multipath environment. 


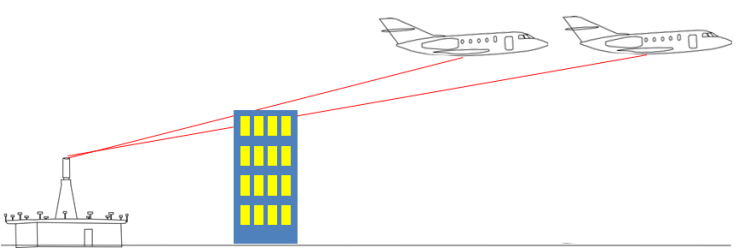

Figure 8: An aircraft flying along a path heading radially away from the ground station, at a fixed altitude. If the line of sight to the ground station is lost, due to occlusion, it is unlikely to be recovered further away from the ground station as the elevation angle becomes smaller.

\section{Background Interference from Com- mercial Providers}

The ISM band is open to unlicensed broadcasting, though some restrictions on power and modulation apply. In the planning phase of the flight trials, we expected non-negligible amounts of background interference. In field tests we did detect a strong background signal in the lower portion of the ISM band that was attributed to a base station for a rural internet service provider (ISP). The ISP operates a ground station located on a water tower, within eyesight of the OU airport, less than $2 \mathrm{~km}$ from our ground set-up.

The signal is pulsed in time, band limited, and relatively high powered compared to the $1 \mathrm{~W}$ OFDM signal broadcast by our setup. Figure 9 shows the time-domain behavior of the pulsed signal over $3 \mathrm{~ms}$, which includes 30 OFDM symbols. The top plot in figure 9 shows the pulsed nature of the interference signal, which has activity gaps in the order of $800 \mu \mathrm{s}$ between bursts. In the frequency domain (bottom plot) we observe that the signal power concentrates in the lower half of the band, leaving us to use $10 \mathrm{MHz}$ in the upper half of the ISM band.

The strategy for leveraging the ISM band for experimentation, in spite of the strong background activity, was to yield to these heavy-duty users; we first yield in frequency, by testing in the $915-925 \mathrm{MHz}$ band, and then in time, by dropping measurements that arrive during a burst of ISM band activity.

To make use of the ISM band signal we discard OFDM symbols that are affected by interference and use only those that are not affected. To detect degraded OFDM symbols we calculate the noise variance within each OFDM symbol after correlation as described in
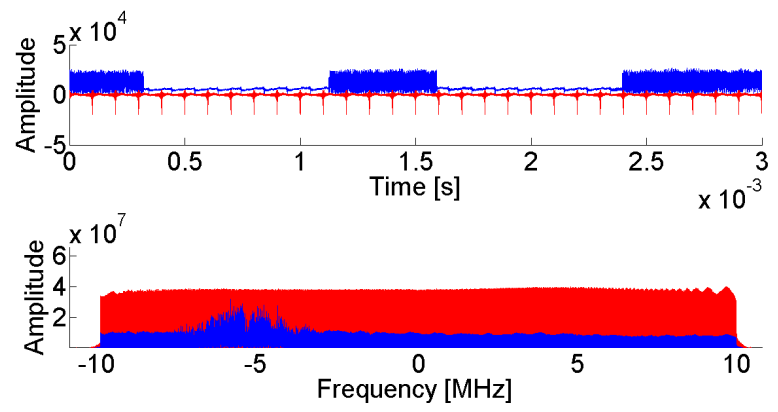

Figure 9: Time and frequency domain behavior of ISM band interference background. For this figure, we measured the band from $905-925 \mathrm{MHz}$. The frequencydomain plot shows strong activity in the lower portion of the spectrum, which was attributed to an internet service provider operating a base station $1.74 \mathrm{~km}$ away from our ground setup, in Albany, OH. Zooming into the upper half of the band, similar behavior can be observed, related to similar services located further away and, therefore, received at a significantly lower power level. The time-domain behavior shows the pulsed nature of the interferer. Depending on the time of the week, the duty cycle of the pulsing could vary significantly, from approximately $30 \%$ (most favorable observed) to approx. $80 \%$.

3. OFDM symbols affected by exhibit a significantly higher noise variance as shown in figure 10 .

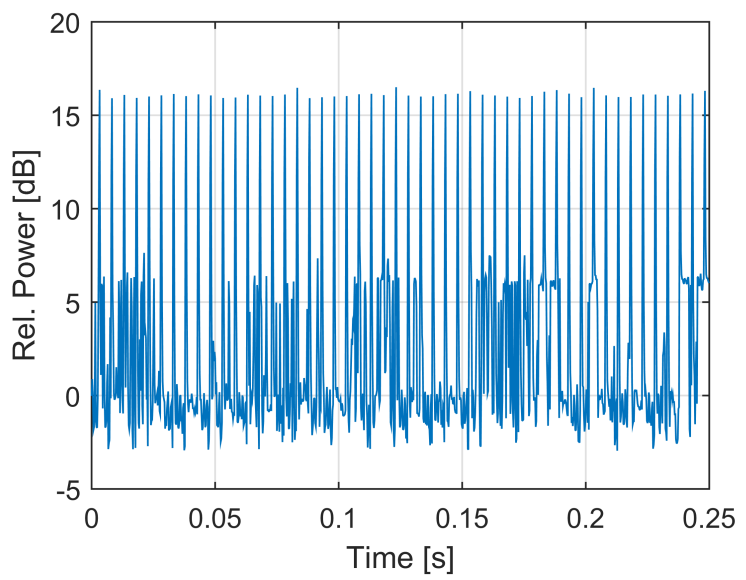

Figure 10: The noise variance of OFDM symbols after correlation indicates background interference. Symbols corrupted by interference are discarded for better estimation of the radio channel.

Figure 10 shows the noise variance during a $250 \mathrm{~ms}$ segment. The figure is normalized to the variance of the white Gaussian background noise. We observe, 


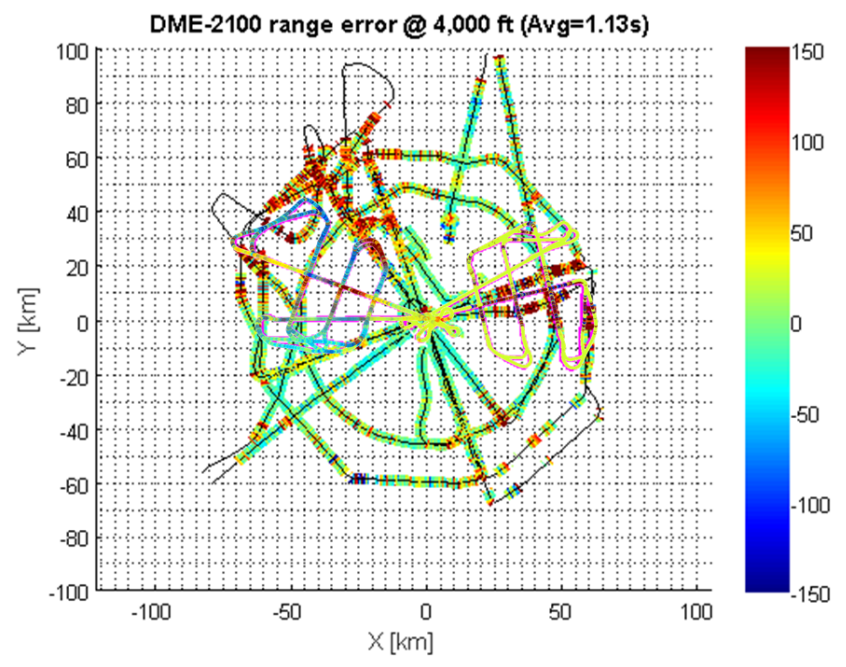

Figure 11: Filling in the predicted DME error at the locations of the two test flights highlights the consistency of the predicted results with the known DME ranging errors.

that after correlation, time segments in which other ISM band users are active can easily be identified. In figure 10 we can clearly identify one user transmitting very short bursts with a repetition cycle of about $200 \mathrm{~Hz}$ which are received at a power level of about $16 \mathrm{~dB}$ relative to the white Gaussian noise power. Another interferer is transmitting with about $6 \mathrm{~dB}$ relative power compared to the noise floor in a more sporadic pattern.

\section{Results}

The main result for this paper is that the predicted DME ranging error is consistent with observations from older testing experiences. The predicted DME ranging errors are mapped onto the paths shown in figure 2 to generate figure 11.

For a better analysis of the consistency between prediction and observation we zoom into one of the two flights and show the detail in figure 12 . In the comparison we observe that the angular sections where strong DME ranging errors are predicted are surrounded by observations of strong DME ranging errors. In particular, the dirty radial is located between two sections where strong ranging errors were observed in the prior flights; furthermore, the distance from the base at which ranging errors start being predicted along the dirty radial is also the distance at which the ranging errors are observed for

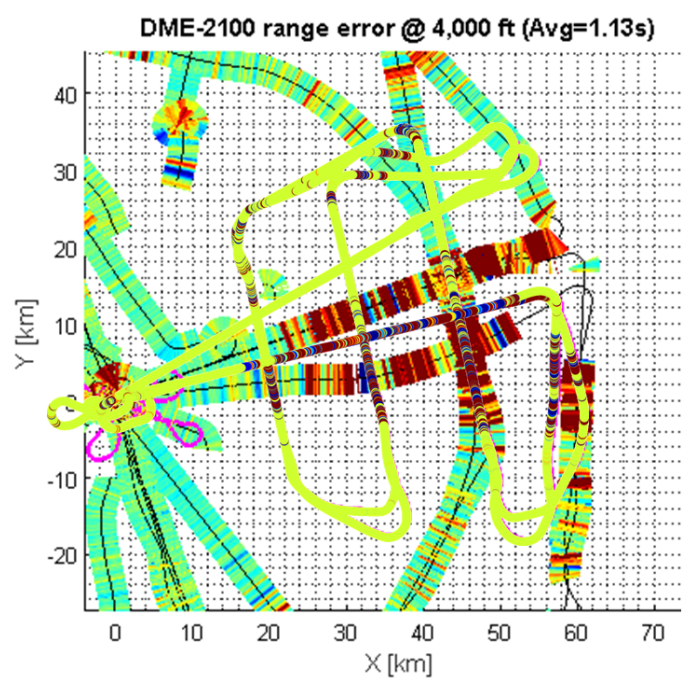

Figure 12: Zooming into one portion of figure 11 helps better appreciate the consistency between prediction and observation.

that angular section.

In addition, the clean radial runs through a portion of the plane where much smaller ranging errors were previously observed. The orbital portions of the predicted error are also consistent with observation, as the strong predicted errors cut off exactly at the same angles as the observed errors do at each orbital segment.

With regards to figure 12 it is important to note that increased ranging errors can occur in clean portions of the map that are not related to the terrain. A typical reason for such occurrences would be the change in the relative orientation of the airborne antenna with respect to the ground, or an occlusion of the LoS by the wings of the aircraft. Further work is needed to adequately model this effect.

\section{Discussion}

An important overall goal on the road to providing integrity from terrestrial ranging sources is understanding multipath-induced ranging errors. The signal processing described above enables an indepth analysis of the received signal, to such a level of detail that multiple propagation paths can be resolved.

From figure 12 we see that strong ranging errors remain in locations where they were identified in prior flight experiments [1, which shows the importance 
of characterizing the spatial correlation of ranging errors. Furthermore, we note that large ranging errors tend to appear where the LoS is occluded and NLOS reception dominates the ranging measurement.

Since occlusion of the LoS is more likely at lower elevations, this phenomenon explains why ranging errors are worst at those points where the flight track is furthest away from the ground station; since the aircraft maintained a constant altitude, the elevation angles at those far-away locations have the lowest elevation angles and, consequently, the strongest ranging errors.

Considering that the results of the flight experiments have so far been congruent with expectations, we see the occlusion or attenuation of the LoS as a critical component of ranging errors from terrestrial sources. Occlusions of the LoS are a consequence of geometric relationships, such as the position of the aircraft, position of the ground station, and position of obstacles; as such, the frequency dependence of occlusions is negligible, compared to the frequency dependence of other ranging error mechanisms. Therefore, we expect the ranging errors for hardware operated in the ISM band to approximate ranging errors for hardware in the ARNS band enough to support prototyping activities.

\section{Future Work}

A topic of great interest is the influence of airframe orientation on ranging errors. In satellite navigation, ranging errors are typically assumed to be a function of the satellite elevation angle at the aircraft position; recent developments suggest that more accurate models could be computed, if relative elevation and azimuth angles are included in the model [7. In a similar fashion, it appears logical to account for the relative orientation between aircraft and ground station, as occlusions of the LoS can be caused by parts of the aircraft and the alignment of the antenna pattern with the LoS can also cause a disturbance of the direct signal.

Another potential line of work is the study of different quantities that correlate well over space and influence the ranging error. It may, for example, be feasible to map scatterers or to compute visibility masks in the vicinity of terrestrial ranging sources. Conversely, a dual problem would be to derive siting criteria with these methods. A map of incidences of ranging errors would certainly be a useful tool in assessing the availability impact of a particular terrestrial ranging source.

\section{Summary}

Assessing the ranging performance of radionavigation systems is essential in providing reliable positioning services. Licensing issues make it cumbersome to run flight experiments with uncertified hardware that uses the ARNS band at 960-1215 MHz. Instead, this work shows that it is possible to test hardware in the nearby ISM band at $902-928 \mathrm{MHz}$ with comparable results. This finding enables a new approach to developing avionics systems, as it simplifies the prototyping and characterization stage. In particular, the new approach is an appealing prospect for the study of multipath-related ranging errors in terrestrial radionavigation systems, as the physical setup could potentially be used for experiments anywhere in ITU Region 2 without requiring any licensing.

\section{References}

[1] Wouter Pelgrum, Kuangmin Li, Adam Naab-Levy, Achim Soelter, George Weida, and Arthur Helwig. eDME On Air: Design, Implementation, and Flight-Test Demonstration. In Proc. of the Int. Techn. Meeting of the ION. Dana Point, CA, pages 40-61, 2015.

[2] Wouter Pelgrum. An Investigation on the Contributing Factors of Enhanced DME Ranging Errors. In Proc. of the Global Nav. Sat. Systems Conf. of the ION. Tampa, FL, 2015.

[3] Nicolas Schneckenburger, Thomas Jost, Dmitriy Shutin, Michael Walter, Thanawat Thiasiriphet, Michael Schnell, and Uwe-carsten Fiebig. Measurement of the L-band air-to-ground channel for positioning applications. IEEE Trans. Aerosp. Electron. Syst. (submitted 2015).

[4] Holger Flühr. Gemeinsame Aspekte der Avionik und der Flugsicherungstechnik. Springer, 2012.

[5] Nicolas Schneckenburger, Dmitriy Shutin, Thomas Jost, Michael Schnell, and Uwe Fiebig. Superresolution Analysis of the L-band Air-to-ground Radio Channel. In Proc. of the Global Nav. Sat. Systems Conf. of the ION. Tampa, FL, 2015.

[6] S. Boyd. Multitone signals with low crest factor. Circuits and Systems, IEEE Transactions on, 33(10):1018-1022, Oct 1986.

[7] Mihaela-Simona Circiu, Michael Felux, Boubeker Belabas, Michael Meurer, Jiyun Lee, Mincham Kim, and Sam Pullen. Evaluation of GPS L5, Galileo E1 and Galileo E5a Performance in Flight Trials for Multi Frequency Multi Constellation GBAS. In Proc. of the Global Nav. Sat. Systems Conf. of the ION. Tampa, FL, 2015. 